\title{
Polymorphisms in Toll-like receptor genes influence antibody responses to cytomegalovirus glycoprotein B vaccine
}

\author{
Ravit Arav-Boger ${ }^{1 *}$, Genevieve L Wojcik ${ }^{2}$, Priya Duggal ${ }^{2}$, Roxann G Ingersoll ${ }^{2}$, Terri Beaty ${ }^{2}$, Robert F Pass ${ }^{3}$ and \\ Robert $\mathrm{H}^{\text {Yolken }}{ }^{1}$
}

\begin{abstract}
Background: Congenital Cytomegalovirus (CMV) infection is an important medical problem that has yet no current solution. A clinical trial of CMV glycoprotein B (gB) vaccine in young women showed promising efficacy. Improved understanding of the basis for prevention of CMV infection is essential for developing improved vaccines.

Results: We genotyped 142 women previously vaccinated with three doses of CMV gB for single nucleotide polymorphisms (SNPS) in TLR 1-4, 6, 7, 9, and 10, and their associated intracellular signaling genes. SNPs in the platelet-derived growth factor receptor (PDGFRA) and integrins were also selected based on their role in binding gB. Specific SNPs in TLR7 and IKBKE (inhibitor of nuclear factor kappa-B kinase subunit epsilon) were associated with antibody responses to gB vaccine. Homozygous carriers of the minor allele at four SNPs in TLR7 showed higher vaccination-induced antibody responses to gB compared to heterozygotes or homozygotes for the common allele. SNP rs1953090 in IKBKE was associated with changes in antibody level from second to third dose of vaccine; homozygotes for the minor allele exhibited lower antibody responses while homozygotes for the major allele showed increased responses over time.
\end{abstract}

Conclusions: These data contribute to our understanding of the immunogenetic mechanisms underlying variations in the immune response to CMV vaccine.

Keywords: Cytomegalovirus, Toll-like receptors, single nucleotide polymorphisms, glycoprotein B vaccine

\section{Background}

Infection with CMV is common in humans, causing severe morbidity and mortality in congenitally-infected newborns and in immunocompromised patients [1-3]. The importance of CMV as the leading infectious cause of mental retardation and deafness in children has been emphasized by its categorization by the Institute of Medicine as a level I vaccine candidate [4]. The rationale for developing a CMV vaccine is based on clinical and animal studies showing that immunity to CMV reduces the frequency and severity of disease $[5,6]$. In addition, animal studies demonstrated that immunization with subunit vaccines prevented disease and transplacental

\footnotetext{
* Correspondence: boger@jhmi.edu

'Department of Pediatrics, Division of Infectious Diseases, Johns Hopkins

Hospital, Baltimore, Maryland 21287-4933, USA

Full list of author information is available at the end of the article
}

transmission of CMV [5-7]. Two recent phase II clinical trials with glycoprotein B (gB)-MF59 led to major enthusiasm and hope for the future success of CMV vaccine. The first was performed in young women recruited on postpartum wards [4], and showed $50 \%$ efficacy in preventing maternal CMV infection. Analysis of antibody levels to $g B$ among vaccine recipients revealed that all women developed antibodies to $\mathrm{gB}$ although the levels and kinetics of antibody responses varied. The second study recruited patients from the kidney/liver transplant waiting list, and showed that antibody titers against $\mathrm{gB}$ were significantly increased one month after the second injection in patients given the vaccine compared with those given placebo, and that antibody titers to gB pretransplant correlated inversely with the duration of viremia, and the need for therapy with ganciclovir after transplant [8].

\section{Biomed Central}


Data from human studies suggest that single nucleotide polymorphisms (SNPs) in immune response genes may influence severity of infections and response to vaccinations such as rubella, measles and hepatitis B [9-16]. Tolllike receptors (TLR) play a key role in the innate immune system and have been implicated in infectious and autoimmune processes [17]. CMV gB and glycoprotein $\mathrm{gH}(\mathrm{gH})$ associate with and activate TLR2/1, mediating an initial signal transduction pathway leading to upregulation of NF-kB and SP-1 $[18,19]$. In liver transplant recipients TLR2 R753Q SNP was associated with CMV replication and disease [20]. The successful gB vaccine trial in young women provided us with a unique opportunity to determine whether antibody responses to $\mathrm{gB}$ vaccine were influenced by SNPs in TLR genes.

\section{Methods}

\section{Study population}

The study cohort included healthy women who were enrolled in the CMV vaccine after obtaining written informed consent [4]. Women were screened on the postpartum wards, and those who were negative for antibody to CMV were invited to participate in the clinical trial. Study participants received a fixed dose of vaccine consisting of recombinant CMV envelope glycoprotein B $(0.02 \mathrm{mg})$ with MF59 adjuvant $(13.25 \mathrm{mg})$. The Johns Hopkins University School of Medicine and University of Alabama Institutional Review Boards granted approval for this study.

\section{Antibody assays}

Antibody to CMV gB was measured using an Enzymelinked immunoabsorbent assay (ELISA) [21]. The vaccine antigen, a recombinant $\mathrm{gB}$ molecule from Towne CMV (provided by Sanofi Pasteur, Marcy L'Etoile, France) was used.

\section{SNP selection}

Using a candidate gene approach the following genes were selected: TLRs and associated intracellular signaling molecules: TLR1-4, TLR6, TLR7, TLR9, TLR10, JUN, MYD88, IKBKE, CHUK (IKK $\alpha$ ), NF-KB1, CD14, MXD3 (MAD3), MAPK8 (JNK1), MAPK14, MAP3K7 (TAK1), LY96 (MD2), TRAF6, IRAK1, IRAK4, TBK1, TICAM1 (TRIF) and IRF3. In addition, PDGFRA, PDGFRB and integrin alpha $\mathrm{V}$ and integrin $\mathrm{B} 1$ were selected based on data showing their role in binding $\mathrm{gB}[22,23]$.

We identified tagging SNPs within the genes and in the $10 \mathrm{~kb}$ flanking each side of the genes, using the LDselect algorithm in individuals from the publicly available HapMap Yoruba, Nigeria population (YRI) [24]. For tagging SNPs we used an $\mathrm{r}^{2}>0.8$. SNPs with a high $\mathrm{r}^{2}$ are correlated and can give information about each other, so they are grouped together and one SNP is selected to represent them all. This is known as a "tagging" SNP. Selection was restricted to those SNPs with a minor allele frequency $(\mathrm{MAF})>1 \%$ in the African population. Additionally, all SNPs were evaluated using a proprietary algorithm from Illumina, Inc. (San Diego, CA) which assesses the likelihood that an assay will succeed on the Illumina genotyping platform. Only those with a design score $>0.8$ were selected for use. For non-synonymous SNPs we used a more liberal cut off score $>0.4$ to ensure inclusion of functional variants. Forty-one additional SNPs with previously reported associations in a variety of human diseases, 157 non-synoymous SNPs, and 28 ancestry informative markers (AIMs) were also typed for a total of 702 SNPs. African Americans are typically individuals with a mixed ethnic background. Allele frequencies of SNPs can vary by race and ethnicity. Therefore, it is important to assess the degree of mixture in an admixed individual when analyzing SNP data. If the cases and controls have differing amounts of admixture, variation in allele frequency by race can cause the appearance of an association with the phenotype of interest, when in reality the association is with race. Including AIMs in the SNP panel allows an assessment of admixture and means that differing amounts of admixture can be controlled for in the data analysis.

\section{Genotyping methods}

Genomic DNA (samples, 75-150 ng/ $\mu \mathrm{l} \mathrm{each}$ ) and 200$400 \mathrm{ng} / \mu \mathrm{l}$ (whole genome amplified DNA each) were obtained from frozen EDTA-blood samples using Gentra Puregene extraction (Qiagen). The study samples and duplicates were plated and genotyped together with 33 HapMap controls (21 CEU; 12 YRI). Genotyping was performed using the Illumina GoldenGate chemistry. Allele cluster definitions for each SNP were initially determined by Illumina's BeadStudio Genotyping Module (version 3.3.7) clustering algorithm using all samples in the project, applying a quality threshold (Gencall score) of 0.25 . This was followed by manual review of the cluster definition for each SNP and adjustment of cluster boundaries to drop questionable genotype calls.

\section{Statistical analysis}

28 ancestry informative markers were genotyped for evaluation of population stratification using principal components (PCA) in the statistical program Eigenstrat [25]. Six HapMap populations from Phase III (CEU, ASW, TSI, YRI, MKK and LWK) were also included. PCA was performed using the 28 AIMs and an additional 55 SNPs from the candidate genes without any linkage disequilibrium (LD). This second analysis did not increase the ability to discriminate between different ethnic groups, so only the results from the first analysis were incorporated into the regression as covariates, 
using the ten first principal components. Association analyses were done in PLINK, version 1.062. A principal component analysis (PCA) was applied to correct for potential confounding due to ancestry. A similar distribution of antibody levels was found among those of European and African ancestry (Additional file 1: Figure S1). In addition, quantile- quantile plots (QQ plots) including lambda values indicated that there was no increased stratification in our study population (Additional file 2: Figure S2). http://pngu.mgh.harvard.edu. ezproxy.welch.jhmi.edu/ purcell/plink/gplink.shtml using linear regression and an additive model. A HardyWeinberg $\mathrm{p}$-value threshold of $10^{-3}$ and a minor allele frequency (MAF) greater than 0.01 were used. A cut off at $p$ value $\leq 0.03$ was selected.

\section{Results}

The study cohort was primarily African American (75\%) with a median age of 19.6 years (range 14-40 years).

SNP data were available from 152 women who received gB vaccine (99\% of attempted samples). Of 152 vaccine recipients, 142 received 3 doses of vaccine and had data available on both genotypes and ELISA titers. The titer of $\mathrm{gB}$ antibodies six month after administration of $3^{\text {rd }}$ vaccine ranged from 370-86,732 (median- 9847.7).

The first goal was to determine whether genetic variation within TLR genes contributed to the observed antibody responses to $\mathrm{CMV} \mathrm{gB}$ vaccine. Linear regression was performed of individuals' log transformed ELISA levels measured 6 months after the third dose of vaccine. SNPs yielding p-value $<0.03$ are summarized in Table 1. Four SNPs in TLR7 (rs179008, rs179009, rs179013 and rs179018) appeared to influence the level of antibodies to gB (Table 1). The linear regression model included potential confounding effects of age, race, educational level, and number of children. Results were similar without adjusting for these covariates (data not shown).

Homozygous carriers of the minor allele at four SNPs in the TLR7 gene showed higher vaccination-induced antibody responses to gB compared to heterozygotes or homozygotes for the common TLR7 allele (Table 1). Three of these four SNPs (rs179009, rs179013, and rs179018) are located in the intronic regions. Rs179008 and rs1790099 are in strong linkage disequilibrium (Figure 1).

Next, a possible association of SNPs in TLRs with changes in antibody responses from one month after the second dose of vaccine to six months following the third (and last) dose was investigated. For this analysis, we used data from 69 women who initially were at the top $50 \%$ of responses (a cut off of 9,506.5 ELISA units was used), and then the change in antibody levels following the last dose of vaccine. Data were compared between women who had no change or an increase in antibody levels, versus those who had a decrease in antibody level. The OR was calculated as the odds of being a weak responder (i.e. having a negative change between visits) versus a strong responder (i.e. having no or positive change) in those who carried the risk allele versus not (Table 2). Two other genes with suggestive association include integrin $\alpha \mathrm{V}$ and MAPK8. Rs1953090 appears to affect changes in antibody level with subsequent immunization with homozygous for the minor allele exhibiting lower antibody levels while homozygotes for the major allele showing increased responses.

\section{Discussion}

Our analysis suggests that polymorphism in TLR genes may influence antibody responses to $\mathrm{CMV}$ gB vaccine. Specifically, SNPs in TLR7 and IKBKE may influence these responses. Viral nucleic acids are a major class of pathogen-associated molecular patterns recognized by TLRs. TLR3 (dsRNA), TLR7 (ssRNA), TLR8 (ssRNA), and TLR9 (CPG DNA) signal from the endosome where degradation of virus particles exposes the viral genome. Several adapter proteins interact with the TLRs: MyD88 (TLR1, 2, 5, 6, 7, 8, and 9), Mal (or Tirap) (TLR2 and 4) and TRIF (TLR3). These adapters link TLR proteins to a downstream signaling pathway including IRAK-1, IRAK-2, TRAF6, and IKK- $\alpha \beta \gamma$. The primary consequences of TLR activation include NF- $\kappa$ B activation,

Table 1 Associations between SNPs in TLRs and gB-Specific Antibody Responses After 3 doses of CMV vaccine

\begin{tabular}{lllllllll}
\hline Gene & SNP & Location & Minor Allele & Counts* $^{*}$ & $\boldsymbol{\beta}$ & Standard Error & P value $^{\text {Median ELISA** }}$ \\
\hline TLR7 & rs179009 & intron & $\mathbf{C}$ & $4 / 47 / 91$ & 0.386 & 0.116 & 0.001 & $21102 / 13194 / 9769$ \\
TLR7 & rs179008 & coding & $T$ & $4 / 34 / 104$ & 0.304 & 0.128 & 0.019 & $21102 / 12907 / 10340$ \\
TLR7 & rs179018 & intron & $G$ & $4 / 36 / 102$ & 0.318 & 0.130 & 0.016 & $16610 / 12907 / 10691$ \\
TLR7 & rs179013 & intron & $T$ & $3 / 34 / 105$ & 0.315 & 0.136 & 0.022 & $22940 / 13501 / 10530$ \\
IKBKE & rs1953090 & intron & $C$ & $15 / 62 / 64$ & -0.275 & 0.097 & 0.005 & $10904 / 9676 / 14993$ \\
TLR3 & rs3775292 & intron & $G$ & $5 / 40 / 96$ & 0.296 & 0.117 & 0.012 & $10904 / 13685 / 9662$ \\
IRAK4 & rs1838341 & intron & $G$ & $0 / 16 / 126$ & -0.458 & 0.204 & 0.027 & $N A / 6394 / 11378$ \\
TLR9 & rs5743849 & intron & $T$ & $0 / 16 / 126$ & -0.469 & 0.211 & 0.028 & $N A / 6635 / 11424$ \\
\hline
\end{tabular}

*Counts are presented as the number of women homozygous for the minor allele/heterozygous/homozygous for the major allele

**Median ELISA- the median gB antibody titer measured in each of the three groups 


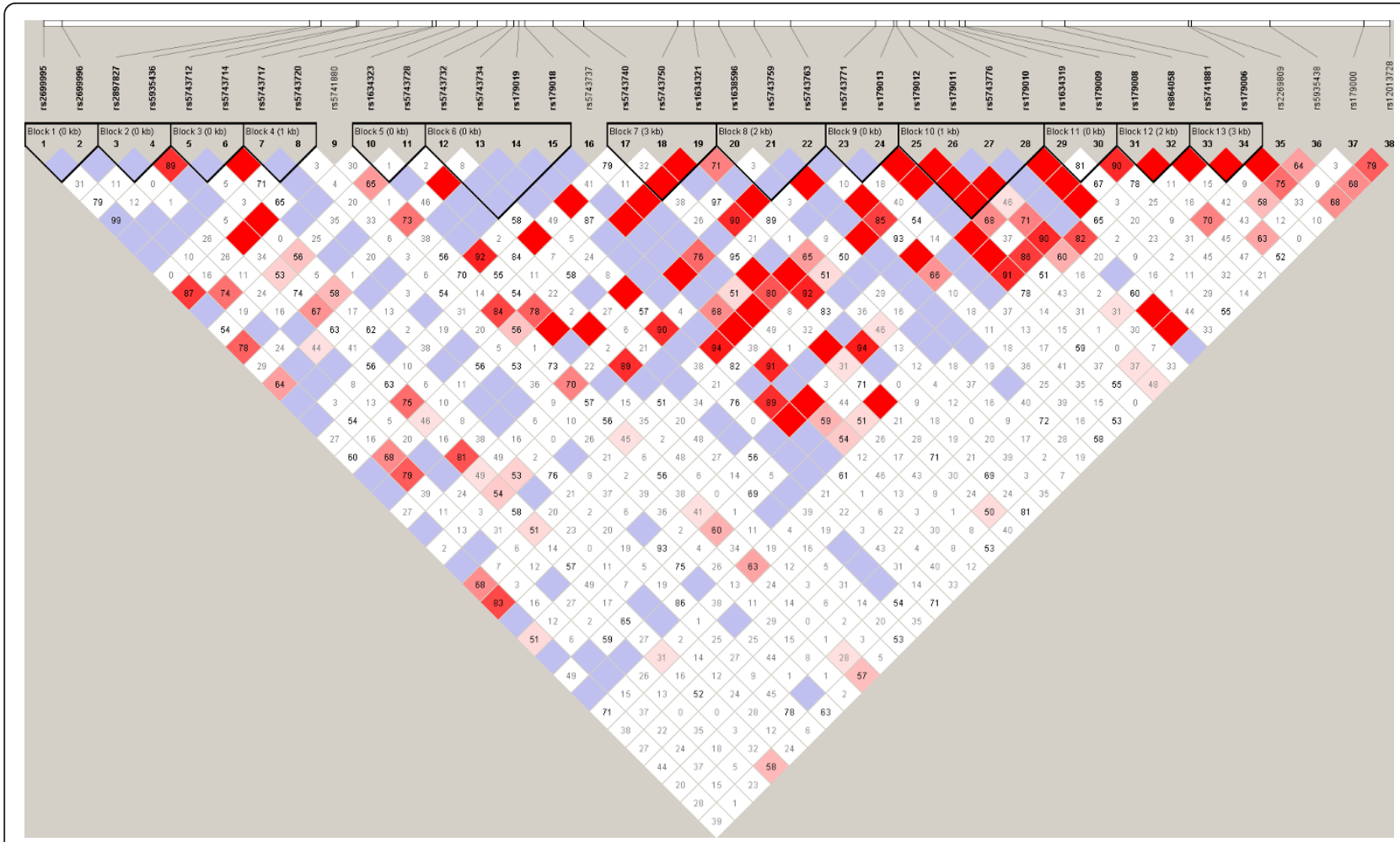

Figure 1 LD Plot of SNPs in TLR7. The blocks are defined by the black triangles using a solid spine of LD in Haploview. The color gradient refers to the degree of linkage disequilibrium, with red indicating strong $L D\left(D^{\prime}\right.$ is high) and white indicating low $L D$ ( $D^{\prime}$ is low). Blue coloring indicates less confidence in the measurement. The actual LD is indicated in the box on a scale of 0-1.

inflammatory cytokine secretion, up-regulation of immune co-stimulatory molecules and, for a subset of TLRs, the production of type I interferon.

Herpesviruses activate signaling through TLR2 and TLR9 [26-28]. TLRs 2, 3, and 9 are involved in innate immune responses to mouse CMV infection $[29,30]$. Studies of human CMV have shown that both $\mathrm{gB}$ and $\mathrm{gH}$ activate TLR2 and physically associate with TLR2 and TLR1 [18]. TLR2 and CD14 recognize CMV virions, and cells exposed to soluble forms of gB activate NF-kB and type I interferon [31-33]. CMV gB and gH mediate an initial signal transduction pathway leading to upregulation of NF-kB and SP-1, an effect which is inhibited by neutralizing antibodies to $\mathrm{gB}$ and $\mathrm{gH}[19]$. A specific singlenucleotide polymorphism (SNP) in TLR2 was associated with CMV replication and disease in a cohort of 92 liver transplant recipients [20].
Surprisingly, we found that specific SNPs in TLR7 were associated with variability in $\mathrm{gB}$ antibodies. SNP rs179008 is associated with amino acid change in position 11 of the TLR7 protein from glutamine to leucine. Several recent studies provide support for a functional effect of this SNP [34,35]. TLR7 is generally considered to function as an intracellular receptor for viral RNAs, but recent data suggest TLR7 exhibits a much broader range of specificities and can interact with endogenous RNAs, as well as bind directly to bacterial pathogens [36,37]. Among viruses, Epstein Barr virus (EBV), a member of the Herpesvirus family, interacts with the TLR7 pathway and enhances B cell proliferation [38]. The role of TLR7 in the immune response to CMV has been suggested in a study that used plasmacytoid dendritic cells (PDCs), the main producers of type I IFN in response to viral infection. Although PDCs were not permissive to

Table 2 Associations between SNPs in TLRs and Changes in Antibody Responses between Second and Third Dose of CMV Vaccine

\begin{tabular}{|c|c|c|c|c|c|}
\hline GENE & SNP ID & Location & Genotype Counts & Fisher's p- value & Median ELISA \\
\hline IKBKE & rs1953090 & intron & $7 / 30 / 31$ & 0.0009319 & $-14103 /-2881 / 3047$ \\
\hline ITGAV & rs3795865 & intron & $1 / 27 / 41$ & 0.0123 & $-36266 /-3084 / 1821$ \\
\hline MAPK8 & rs17010454 & intron & $0 / 8 / 61$ & 0.02247 & 0/2862/-1708 \\
\hline
\end{tabular}


CMV infection, they secreted cytokines after contact with $\mathrm{CMV}$, including IFN $-\alpha$ secretion that was blocked by inhibition of TLR7 and TLR9, suggesting an engagement of the TLR7 and/or TLR9 pathways. Through PDC stimulation, CMV prompted B cell activation, but only induced antibody production in the presence of $\mathrm{T}$ cells [39].

The best known TLR member expressed in human B cells is TLR9 [40], but other TLRs including TLR7 are also expressed. TLR7 ligands activate murine B cells [41] but TLR7 responsiveness of naive human $B$ cells is controlled by PDC [42]. IFN- $\alpha$ released from PDCs upon stimulation with TLR7 or TLR9 ligands was found to be responsible for increased TLR7 sensitivity of B cells. Depletion of PDC from mixed cell cultures (PBMC) lead to a complete loss of TLR7 ligand-induced B cell proliferation. Our data suggest that TLR7 could interact with one of the components of the $\mathrm{gB}$ vaccine, or that vaccination induces upregulation of endogenous RNAs subsequently recognized by TLR7. Activation of TLR7 upon gB immunization may induce B-cells to proliferate and generate antibodies. While the MF59 adjuvant could also play a role in TLR7 activation, a recent study showed that MF59 did not activate any of the TLRs in vitro [43]. We note that activation via TLR pathways is not essential for the function of every adjuvant [44]. Alum adjuvant, as well as complete and incomplete Freund's adjuvants, enhanced antibody responses in mice genetically-engineered to be unable to signal through the major TLR adapters MyD88.

IKBKE, a noncanonical I-kappa-B kinase (IKK), is essential for regulating antiviral signaling pathways. It phosphorylates inhibitors of NF-kappa-B, leading to dissociation of the inhibitor/NF-kappa-B complex and ultimately degradation of the inhibitor. IKBKE is expressed predominantly in immune cells and tissues, including peripheral blood leukocytes, spleen, and thymus [45]. IKBKE phosphorylates IRF-3 and is required for its full activation in CMV-infected smooth muscle cells [46]. Thus, it has a pivotal role in coordinating the activation of IRF3 and NF-kB in the innate immune response. Although IRF-3 was initially reported to be activated by recombinant gB [47], recently Z-DNA binding protein 1 , ZBP1 was suggested as an essential gene for IRF3 activation by CMV [48]. A recent study demonstrated that induction of inflammatory cytokines by CMV was mediated via a TLR-2-dependent activation of NF- $\kappa$ B [31], but it did not specifically evaluate whether IRF-3 was activated in a TLR-2-dependent fashion by CMV. So far, the literature has only shown TLR-3 and TLR-4 can induce type I interferon production through IRF-3 activation. The ability of TLR-7 to induce type I interferon through IRF-3 has not been studied yet.

\section{Conclusions}

This study shows for the first time that host genetics plays a role in the development of $\mathrm{gB}$ antibodies following CMV immunization. It is unclear whether these polymorphisms contribute to protective immunity. Future studies will provide additional understanding of the roles of immune response genes in CMV immunization and will evaluate the role of $\mathrm{gB}$ in activation of TLR7-related pathways.

\section{Availability of supporting data}

The data set supporting the results of this article is included within the article

\section{Additional material}

Additional file 1: Figure S1. SNPs in ancestry informative markers and antibody levels in the study population. A principal component analysis was performed to correct for potential confounding due to ancestry. The distribution of antibody levels is depicted for African and European women.

Additional file 2: Figure S2. Quantile-Quantile (QQ) plots of the log antibody levels QQ plots of log antibody levels and lambda values are depicted. In the right panel, nothing is done to account for the structure. On the left panel, the results are adjusted for principal components, leaving about the same amount of inflation as the case with no population stratification.

\section{Abbreviations}

CMV: Cytomegalovirus; gB: glycoprotein B; TLR: Toll-like receptors; PDGFR: Platelet derived growth factor receptor; IKBKE: Inhibitor of nuclear factor kappa-B kinase subunit epsilon; SNP: Single nucleotide polymorphism

\section{Acknowledgements}

Supported by the Johns Hopkins Institute of Clinical and Translational Research and the National Institutes of health [KO8-AI074907] to RAB and U01-Al063565 to RFP. Genotyping services were provided by the Johns Hopkins University under federal contract number [NO1-HV-48195] from the National Heart, Lung, and Blood Institute.

\section{Author details}

${ }^{1}$ Department of Pediatrics, Division of Infectious Diseases, Johns Hopkins Hospital, Baltimore, Maryland 21287-4933, USA. ${ }^{2}$ Department of Epidemiology Johns Hopkins Bloomberg School of Public Health, Baltimore, MD, 21231-1000, USA. ${ }^{3}$ Department of Pediatrics, Division of Infectious Diseases, University of Alabama at Birmingham, Birmingham, Alabama 35294, USA.

\section{Authors' contributions}

RAB conceived of the study, its design and wrote the manuscript; GLW, PD and TB performed and reviewed the statistical analysis, RGI helped in SNP selection, RFP provided essential samples and edited the manuscript, RHY provided input into experimental design. All authors read and approved the final manuscript.

\section{Competing interests}

Ravit Arav-Boger received research funding from Sanofi-Pasteur. Robert F Pass receives research funding from Sanofi-Pasteur. In addition, Dr. Pass has served as a consultant to Merck, Vical, AlphaVax and Medlmmune. Other authors: no conflict.

Received: 12 December 2011 Accepted: 13 March 2012 Published: 13 March 2012 


\section{References}

1. Kenneson A, Cannon MJ: Review and meta-analysis of the epidemiology of congenital cytomegalovirus (CMV) infection. Rev Med Virol 2007, 17:253-276.

2. Yow MD, Demmler GJ: Congenital cytomegalovirus disease-20 years is long enough. N Engl J Med 1992, 326:702-703.

3. Griffiths PD, Clark DA, Emery VC: Betaherpesviruses in transplant recipients. J Antimicrob Chemother 2000, 45(Suppl T3):29-34.

4. Pass RF, Zhang C, Evans A, Simpson T, Andrews W, Huang ML, Corey L, Hill J, Davis E, Flanigan C, Cloud G: Vaccine prevention of maternal cytomegalovirus infection. N Engl J Med 2009, 360:1191-1199.

5. Fowler KB, Stagno S, Pass RF: Maternal immunity and prevention of congenital cytomegalovirus infection. JAMA 2003, 289:1008-1011.

6. Schleiss MR, Bourne N, Bernstein DI: Preconception vaccination with a glycoprotein $\mathrm{B}(\mathrm{gB})$ DNA vaccine protects against cytomegalovirus (CMV) transmission in the guinea pig model of congenital CMV infection. J Infect Dis 2003, 188:1868-1874.

7. Schleiss MR, Lacayo JC, Belkaid Y, McGregor A, Stroup G, Rayner J, Alterson K, Chulay JD, Smith JF: Preconceptual administration of an alphavirus replicon UL83 (pp 65 homolog) vaccine induces humoral and cellular immunity and improves pregnancy outcome in the guinea pig model of congenital cytomegalovirus infection. J Infect Dis 2007, 195:789-798.

8. Griffiths PD, Stanton A, McCarrell E, Smith C, Osman M, Harber M, Davenport A, Jones G, Wheeler DC, O'Beirne J, Thorburn D, Patch D, Atkinson CE, Pichon S, Sweny P, Lanzman M, Woodford E, Rothwell E, Old N, Kinyanjui R, Haque T, Atabani S, Luck S, Prideaux S, Milne RS, Emery VC, Burroughs AK: Cytomegalovirus glycoprotein-B vaccine with MF59 adjuvant in transplant recipients: a phase 2 randomised placebocontrolled trial. Lancet 2011, 377:1256-1263.

9. Kurt-Jones EA, Popova L, Kwinn L, Haynes LM, Jones LP, Tripp RA, Walsh EE, Freeman MW, Golenbock DT, Anderson LJ, Finberg RW: Pattern recognition receptors TLR4 and CD14 mediate response to respiratory syncytial virus. Nat Immunol 2000, 1:398-401.

10. Bieback K, Lien E, Klagge IM, Avota E, Schneider-Schaulies J, Duprex WP, Wagner H, Kirschning CJ, Ter MV, Schneider-Schaulies S: Hemagglutinin protein of wild-type measles virus activates toll-like receptor 2 signaling. J Virol 2002, 76:8729-8736.

11. Poland GA: Variability in immune response to pathogens: using measles vaccine to probe immunogenetic determinants of response. Am J Hum Genet 1998, 62:215-220.

12. Poland GA, Jacobson RM: The genetic basis for variation in antibody response to vaccines. Curr Opin Pediatr 1998, 10:208-215.

13. Hohler T, Reuss E, Evers N, Dietrich E, Rittner C, Freitag CM, Vollmar J, Schneider PM, Fimmers R: Differential genetic determination of immune responsiveness to hepatitis $B$ surface antigen and to hepatitis $A$ virus: $a$ vaccination study in twins. Lancet 2002, 360:991-995.

14. Wang C, Tang J, Song W, Lobashevsky E, Wilson CM, Kaslow RA: HLA and cytokine gene polymorphisms are independently associated with responses to hepatitis B vaccination. Hepatology 2004, 39:978-988.

15. Desombere I, Hauser P, Rossau R, Paradijs J, Leroux-Roels G: Nonresponders to hepatitis B vaccine can present envelope particles to $T$ lymphocytes. J Immunol 1995, 154:520-529.

16. Pankratz VS, Vierkant RA, O'Byrne MM, Ovsyannikova IG, Poland GA Associations between SNPs in candidate immune-relevant genes and rubella antibody levels: a multigenic assessment. BMC Immunol 2010, 11:48.

17. Green NM, Marshak-Rothstein A: Toll-like receptor driven B cell activation in the induction of systemic autoimmunity. Semin Immunol 2011, 23:106-112.

18. Boehme KW, Guerrero M, Compton T: Human cytomegalovirus envelope glycoproteins $\mathrm{B}$ and $\mathrm{H}$ are necessary for TLR2 activation in permissive cells. J Immunol 2006, 177:7094-7102.

19. Yurochko AD, Hwang ES, Rasmussen L, Keay S, Pereira L, Huang ES: The human cytomegalovirus UL55 (gB) and UL75 (gH) glycoprotein ligands initiate the rapid activation of Sp1 and NF-kappaB during infection. $J$ Virol 1997, 71:5051-5059.

20. Kijpittayarit S, Eid AJ, Brown RA, Paya CV, Razonable RR: Relationship between Toll-like receptor 2 polymorphism and cytomegalovirus disease after liver transplantation. Clin Infect Dis 2007, 44:1315-1320.
21. Hackett DJ, Zhang C, Stefanescu C, Pass RF: Enzyme-linked immunosorbent assay for measurement of cytomegalovirus glycoprotein B antibody in serum. Clin Vaccine Immunol 2010, 17:836-839.

22. Soroceanu L, Akhavan A, Cobbs CS: Platelet-derived growth factor-alpha receptor activation is required for human cytomegalovirus infection. Nature 2008, 455:391-395.

23. Wang $X$, Huang DY, Huong SM, Huang ES: Integrin alphavbeta3 is a coreceptor for human cytomegalovirus. Nat Med 2005, 11:515-521.

24. Howie BN, Carlson CS, Rieder MJ, Nickerson DA: Efficient selection of tagging single-nucleotide polymorphisms in multiple populations. Hum Genet 2006, 120:58-68.

25. Price AL, Patterson NJ, Plenge RM, Weinblatt ME, Shadick NA, Reich D: Principal components analysis corrects for stratification in genome-wide association studies. Nat Genet 2006, 38:904-909.

26. Finberg RW, Knipe DM, Kurt-Jones EA: Herpes simplex virus and toll-like receptors. Viral Immunol 2005, 18:457-465.

27. Kurt-Jones EA, Belko J, Yu C, Newburger PE, Wang J, Chan M, Knipe DM, Finberg RW: The role of toll-like receptors in herpes simplex infection in neonates. J Infect Dis 2005, 191:746-748.

28. Ashkar AA, Yao XD, Gill N, Sajic D, Patrick AJ, Rosenthal KL: Toll-like receptor (TLR)-3, but not TLR4, agonist protects against genital herpes infection in the absence of inflammation seen with CpG DNA. J Infect Dis 2004, 190:1841-1849.

29. Tabeta K, Georgel P, Janssen E, Du X, Hoebe K, Crozat K, Mudd S, Shamel L, Sovath S, Goode J, Alexopoulou L, Flavell RA, Beutler B: Toll-like receptors 9 and 3 as essential components of innate immune defense against mouse cytomegalovirus infection. Proc Natl Acad Sci USA 2004, 101:3516-3521.

30. Szomolanyi-Tsuda E, Liang X, Welsh RM, Kurt-Jones EA, Finberg RW: Role for TLR2 in NK cell-mediated control of murine cytomegalovirus in vivo. J Virol 2006, 80:4286-4291.

31. Compton T, Kurt-Jones EA, Boehme KW, Belko J, Latz E, Golenbock DT, Finberg RW: Human cytomegalovirus activates inflammatory cytokine responses via CD14 and Toll-like receptor 2. J Virol 2003, 77:4588-4596.

32. Simmen KA, Singh J, Luukkonen BG, Lopper M, Bittner A, Miller NE, Jackson MR, Compton T, Fruh K: Global modulation of cellular transcription by human cytomegalovirus is initiated by viral glycoprotein B. Proc Natl Acad Sci USA 2001, 98:7140-7145.

33. Boyle KA, Pietropaolo RL, Compton T: Engagement of the cellular receptor for glycoprotein B of human cytomegalovirus activates the interferonresponsive pathway. Mol Cell Biol 1999, 19:3607-3613.

34. Askar E, Ramadori G, Mihm S: Toll-like receptor 7 rs179008/GIn11Leu gene variants in chronic hepatitis C virus infection. J Med Virol 2010, 82:1859-1868.

35. Edwards AO, Chen D, Fridley BL, James KM, Wu Y, Abecasis G, Swaroop A Othman M, Branham K, lyengar SK, Sivakumaran TA, Klein R, Klein BE, Tosakulwong N: Toll-like receptor polymorphisms and age-related macular degeneration. Invest Ophthalmol Vis Sci 2008, 49:1652-1659.

36. Boehme KW, Singh J, Perry ST, Compton T: Human cytomegalovirus elicits a coordinated cellular antiviral response via envelope glycoprotein B. Virol 2004, 78:1202-1211.

37. DeFilippis VR, Alvarado D, Sali T, Rothenburg S, Fruh K: Human cytomegalovirus induces the interferon response via the DNA sensor ZBP1. J Virol 2010, 84:585-598.

38. Martin HJ, Lee JM, Walls D, Hayward SD: Manipulation of the toll-like receptor 7 signaling pathway by Epstein-Barr virus. J Virol 2007, 81:9748-9758.

39. Varani S, Cederarv M, Feld S, Tammik C, Frascaroli G, Landini MP, Soderberg-Naucler C: Human cytomegalovirus differentially controls B cell and $\mathrm{T}$ cell responses through effects on plasmacytoid dendritic cells. $\mathrm{J}$ Immunol 2007, 179:7767-7776.

40. Hornung V, Rothenfusser S, Britsch S, Krug A, Jahrsdorfer B, Giese T, Endres S, Hartmann G: Quantitative expression of toll-like receptor 1-10 mRNA in cellular subsets of human peripheral blood mononuclear cells and sensitivity to CpG oligodeoxynucleotides. J Immunol 2002, 168:4531-4537.

41. Tomai MA, Imbertson LM, Stanczak TL, Tygrett LT, Waldschmidt TJ: The immune response modifiers imiquimod and R-848 are potent activators of B lymphocytes. Cell Immunol 2000, 203:55-65. 
42. Bekeredjian-Ding IB, Wagner M, Hornung V, Giese T, Schnurr M, Endres $S$, Hartmann G: Plasmacytoid dendritic cells control TLR7 sensitivity of naive B cells via type I IFN. J Immunol 2005, 174:4043-4050.

43. Seubert A, Calabro S, Santini L, Galli B, Genovese A, Valentini S, Aprea S, Colaprico A, D'Oro U, Giuliani MM, Pallaoro M, Pizza M, O'Hagan DT, Wack A, Rappuoli R, De GE: Adjuvanticity of the oil-in-water emulsion MF59 is independent of Nlrp3 inflammasome but requires the adaptor protein MyD88. Proc Natl Acad Sci USA 2011, 108:11169-11174.

44. Gavin AL, Hoebe K, Duong B, Ota T, Martin C, Beutler B, Nemazee D: Adjuvant-enhanced antibody responses in the absence of toll-like receptor signaling. Science 2006, 314:1936-1938.

45. Gravel SP, Servant MJ: Roles of an IkappaB kinase-related pathway in human cytomegalovirus-infected vascular smooth muscle cells: a molecular link in pathogen-induced proatherosclerotic conditions. J Biol Chem 2005, 280:7477-7486.

46. Fitzgerald KA, McWhirter SM, Faia KL, Rowe DC, Latz E, Golenbock DT, Coyle AJ, Liao SM, Maniatis T: IKKepsilon and TBK1 are essential components of the IRF3 signaling pathway. Nat Immunol 2003, 4:491-496.

47. Boehme KW, Singh J, Perry ST, Compton T: Human cytomegalovirus elicits a coordinated cellular antiviral response via envelope glycoprotein B. J Virol 2004, 78:1202-1211.

48. DeFilippis VR, Alvarado D, Sali T, Rothenburg S, Fruh K: Human cytomegalovirus induces the interferon response via the DNA sensor ZBP1. J Virol 2010, 84:585-598.

doi:10.1186/1756-0500-5-140

Cite this article as: Arav-Boger et al: Polymorphisms in Toll-like receptor genes influence antibody responses to cytomegalovirus glycoprotein B vaccine. BMC Research Notes 2012 5:140.

\section{Submit your next manuscript to BioMed Central and take full advantage of:}

- Convenient online submission

- Thorough peer review

- No space constraints or color figure charges

- Immediate publication on acceptance

- Inclusion in PubMed, CAS, Scopus and Google Scholar

- Research which is freely available for redistribution

Submit your manuscript at www.biomedcentral.com/submit 